\title{
Geometry and Dynamics of Quantum State Diffusion
}

\author{
Nikola Burić * \\ Institute of Physics, P.O. Box 57, 11001 Belgrade, Serbia.
}

November 26, 2018

\begin{abstract}
Riemannian metric on real $2 \mathrm{n}$-dimensional space associated with the equation governing complex diffusion of pure states of an open quantum system is introduced and studied. Examples of a qubit under the influence of dephasing and thermal environments are used to show that the curvature of the diffusion metric is a good indicator of the properties of the environment dominated evolution and its stability.
\end{abstract}

PACS: 03.65.Yz

*e-mail: buric@phy.bg.ac.yu 


\section{Introduction}

The states of an open quantum system are commonly described by a density matrix $\hat{\rho}$. In many cases, the evolution of $\hat{\rho}(t)$ is governed by a master equation of the Linblad form [1, [2] for the density matrix $\hat{\rho}(t)$

$$
\frac{d \hat{\rho}(t)}{d t}=-i[\hat{H}, \hat{\rho}]+\frac{1}{2} \sum_{l}\left[\hat{L}_{l} \hat{\rho}, \hat{L}_{l}^{\dagger}\right]+\left[\hat{L}_{l}, \hat{\rho} \hat{L}_{l}^{\dagger}\right]
$$

where the Linblad operators $\hat{L}_{l}$ describe the influence of the environment. The equation (1) represents the general form of an evolution equation for a quantum system which satisfies Markov property.

However, this theoretical approach to the dynamics of open quantum systems is not unique. In real experiments it is often useful to understand and model the dynamics of pure quantum states [3], [4], [5]. Indeed, the evolution of an open system can be described directly in terms of the dynamics of the system's pure state. The corresponding evolution equation is a stochastic modification of the unitary Schroedinger equation. In fact, the density matrix $\hat{\rho}$ can be written, in different but equivalent ways, as a convex combination of pure states. Each of these results in a stochastic differential equation for $\mid \psi(t)>$ in the Hilbert space $\mathcal{H}$. Such stochastic Schroedinger equations (SSE) are called stochastic unravelling [6],[7],[2] of the Linblad master equation for the reduced density matrix $\hat{\rho}(t)$. There are many different forms of nonlinear and linear SSE that have been used in the context of open systems [8], [3], [2], [6], [7] or suggested as fundamental modifications of the Schroedinger equation [9] [10,,11], 6] [12,,13]. They are all consistent with the requirement that the solutions of (1) and of SSE satisfy

$$
\hat{\rho}(t)=E[|\psi(t)><\psi(t)|] .
$$

where $E[|\psi(t)><\psi(t)|]$ is the expectation with respect to the distribution of the stochastic process $\mid \psi(t)>$. The advantages of the description in terms of the pure states and SSE over the description by $\hat{\rho}$ are twofold. On the practical side, the computations are much more practical, as soon as the size of the Hilbert space is moderate or large [14. On the theoretical side, the stochastic evolution of pure states provides valuable insides which can not be inferred from the density matrix approach [15],[16], [6], [2], [5], [17].

There are two main approaches to the unravelling of the Linblad master equation: the method of quantum state diffusion [6] and the relative state 
method [3], 22, with specific advantages associated with each of the methods. The relative state method is usually used do describe the situations when the measurement is the dominant interaction with the environment. The method offers particular flexibility in that the master equation can be unravelled into different stochastic equations conditioned on the results of measurement. On the other hand the correspondence between the QSD equations and the Linblad master equations is unique, and is not related to a particular measurement scheme, or the form of the Markov environment. The resulting SSE is always of the form of a diffusion process on the Hilbert space of pure states, which is its main property to be explored in this paper.

We shall concentrate on the unique unravelling of the master equation given by the quantum state diffusion equation, and explore the fact that it represents a diffusion process. QSD equation is the unique unravelling of (1) which preserves the norm of the state vector and has the same invariance as (1) under the unitary transformations of the environment operators $\left\{\hat{L}_{l}\right\},[6]$. The equation is given by the following formula:

$$
\begin{aligned}
\mid d \psi> & =-i \hat{H} \mid \psi>d t \\
& +\left[\sum_{l} 2<\hat{L}_{l}^{\dagger}>\hat{L}_{l}-\hat{L}_{l}^{\dagger} \hat{L}_{l}-<\hat{L}_{l}^{\dagger}><\hat{L}_{l}>\right] \mid \psi(t)>d t \\
& +\sum_{l}\left(\hat{L}_{l}-<L_{l}>\right) \mid \psi(t)>d W_{l}
\end{aligned}
$$

where $\langle>$ denotes the quantum expectation in the state $| \psi(t)>$ and $d W_{l}$ are independent increments (indexed by $l$ ) of complex Wiener c-number processes $W_{l}(t)$.

The equation (3) represent a diffusion process on a complex vector space. We shall utilize the diffusion matrix of this process to define a Riemannian metric on the corresponding real space. We shall then study the properties of this diffusion metric as a field fixed by the environment and in relation to the stochastic evolution of the state vector, for different types of the environment. It will be shown, using examples of the dephasing and thermal environments and the measurement of an observable, that the curvature of the diffusion metric is a good indicator of the properties of the environment dominated evolution and its stability. We shall see that the curvature maxima of the diffusion metric coincide with the states that are preferred by the particular type of the environment. Furthermore, if the maxima are sharp and positive the stochastic dynamics governed by the environment and a Hamiltonian 
perturbation that does not commute with $\hat{L}_{l}$, is likely to be attracted to the state with the maximal (positive) curvature. On the other hand the states that correspond to the negative values of the curvature are unstable. Our analyzes of the QSD equation, and the results, are strictly related to the fact that the equation represent a norm-preserving diffusion process, and in this sense are applicable to the stochastic modifications of the Schroedinger equation that describe a norm-preserving diffusion on the Hilbert space of pure states, like the QSD equation and, for example, the equations of the spontaneous collapse models [13].

The structure of the paper is as follows. We shall first discuss, in the next section, a way to relate a Riemannian metric on a real space $R^{2 n}$ to a complex diffusion process on $C^{n}$. Then, in section 3 , we shall apply this procedure to define the Riemannian metric associated with QSD, and than the properties of this metric for various types of environments will be studied. Finally, in section 4, we shall summarize and discuss our results.

\section{Riemannian metric of a complex diffusion}

Using the following notation

$$
\begin{aligned}
f(\mid \psi>) & =-i \hat{H} \mid \psi> \\
& +\left[\sum_{l} 2<\psi\left|\hat{L}_{l}^{\dagger}\right| \psi>\hat{L}_{l}-\hat{L}_{l}^{\dagger} \hat{L}_{l}-<\psi\left|\hat{L}_{l}^{\dagger}\right| \psi><\psi\left|\hat{L}_{l}\right| \psi>\right] \mid \psi> \\
B(\mid \psi>) d W & =\sum_{l}\left(\hat{L}_{l}-<\psi\left|L_{l}\right| \psi>\right) \mid \psi>d W_{l} .
\end{aligned}
$$

the QSD equation (3) assumes the standard form of a stochastic differential equation (SDE) for an $n$-dimensional autonomous (stationary) complex diffusion process:

$$
d \mid \psi>=f(\mid \psi>) d t+B(\mid \psi>) d W
$$

$\mid \psi(t)>$ and $f(\mid \psi(t)>)$ are complex vectors of complex dimension $n$, and $d W$ are differential increments of an m-dimensional complex Wiener process:

$$
\begin{aligned}
\mathrm{E}\left[d W_{l}\right]=\mathrm{E}\left[d W_{l} d W_{l^{\prime}}\right] & =0 \\
\mathrm{E}\left[d W_{l} d \bar{W}_{l^{\prime}}\right] & =\delta_{l, l^{\prime}} d t \\
l & =1,2 \ldots m
\end{aligned}
$$


where $E[\cdot]$ denotes the expectation with respect to the probability distribution given by the (m-dimensional) process $W$, and $\bar{W}_{l}$ is the complex conjugate of $W_{l} . B(\mid \psi>)$ is $n \times m$ matrix, where $m$ is at most $n^{2}-1$, and the diffusion matrix is

$$
G=B B^{\dagger}
$$

Thus, $G(|\psi\rangle)$ is Hermitian and nonnegative-definite. Notice that, unlike the case of a general SDE, the dissipative part of the drift (4) and the diffusion term (5) are determined by the same operators $\hat{L}_{l}$, and related in such a way that the diffusion equation preserves the norm of the state vector.

The complex n-dimensional equation (3) generates 2 n-dimensional real diffusion. Let us introduce the following real $n$ dimensional vectors

$$
\begin{aligned}
p & =\frac{i}{\sqrt{2}}(\bar{\psi}-\psi), & q & =\frac{1}{\sqrt{2}}(\bar{\psi}+\psi) \\
\psi & =\frac{1}{\sqrt{2}}(q+i p), & \bar{\psi} & =\frac{1}{\sqrt{2}}(q-i p),
\end{aligned}
$$

and a $2 n$ dimensional vector $X=(q, p)$. Similarly, we introduce real and imaginary parts of the vector $f$ and order them as components of a $2 \mathrm{n}$ real vector $\mathcal{F}=\left(f^{R}, f^{I}\right)$, and introduce real and imaginary parts of the increments of the complex m-dim Wiener process $d W$ by

$$
d W_{i}=\left(d W_{i}^{R}+i d W_{i}^{I}\right) / \sqrt{2}, i=1,2, \ldots m
$$

It is easily checked that the real and the imaginary parts are increments of a real $2 \mathrm{~m}$-dimensional process, i.e.

$$
E\left(d W_{i}^{R} d W_{j}^{R}\right)=E\left(d W_{i}^{I} d W_{j}^{I}\right)=\delta_{i, j} d t, \quad E\left(d W_{i}^{R} d W_{j}^{I}\right)=0 .
$$

With this notation we have

$$
\left(\begin{array}{c}
d q \\
d p
\end{array}\right)=\frac{1}{\sqrt{2}}\left(\begin{array}{c}
d \bar{\psi}+d \psi \\
d \bar{\psi}-d \psi
\end{array}\right)
$$

Substitution of the complex equation (3) and its complex conjugate, leads to the following $2 n$ dimensional real SDE:

$$
\left(\begin{array}{l}
d q \\
d p
\end{array}\right)=\left(\begin{array}{c}
f^{R}(p, g) \\
f^{I}(p, q)
\end{array}\right)+\frac{1}{\sqrt{2}}\left(\begin{array}{cc}
B^{R} & -B^{I} \\
B^{I} & B^{R}
\end{array}\right)\left(\begin{array}{c}
d W^{R} \\
d W^{I}
\end{array}\right),
$$


The matrix $\mathcal{B}$ of dimension $2 n \times 2 m$

$$
\mathcal{B}=\frac{1}{\sqrt{2}}\left(\begin{array}{cc}
B^{R} & -B^{I} \\
B^{I} & B^{R}
\end{array}\right)
$$

where

$$
(B)_{i j}=\left(B^{R}\right)_{i j}+i\left(B^{I}\right)_{i j}
$$

gives the diffusion matrix $\mathcal{G}$ for the real $2 n$ dimensional diffusion described by the process (13)

$$
\mathcal{G}=\mathcal{B B}^{T}=\frac{1}{2}\left(\begin{array}{cc}
\left(B^{R}\right)\left(B^{R}\right)^{T}+\left(B^{I}\right)\left(B^{I}\right)^{T} & \left(B^{R}\right)\left(B^{I}\right)^{T}-\left(B^{I}\right)\left(B^{R}\right)^{T} \\
\left(B^{I}\right)\left(B^{R}\right)^{T}-\left(B^{R}\right)\left(B^{I}\right)^{T} & \left(B^{I}\right)\left(B^{I}\right)^{T}+\left(B^{R}\right)\left(B^{R}\right)^{T}
\end{array}\right)
$$

We can write the matrix $\mathcal{G}$ in terms of real and imaginary components of the $n \times n$ complex matrix $G=B B^{\dagger}$ as follows

$$
\mathcal{G}=\frac{1}{2}\left(\begin{array}{cc}
G^{R} & G^{I} \\
-G^{I} & G^{R}
\end{array}\right)=\frac{1}{2}\left[\left(\begin{array}{cc}
G^{R} & 0 \\
0 & G^{R}
\end{array}\right)+\left(\begin{array}{cc}
0 & -1 \\
1 & 0
\end{array}\right)\left(\begin{array}{cc}
G^{I} & 0 \\
0 & G^{I}
\end{array}\right)\right]
$$

where $-G^{I}=\left(G^{I}\right)^{T}$, since the matrix $G$ is Hermitian. Furthermore, one can see that, besides the equalities between the entries corresponding to the symmetry of the matrix, there are other equalities

$$
(\mathcal{G})_{i, j}=(\mathcal{G})_{i+n, j+n}, \quad i, j=1,2 \ldots n
$$

The matrix $\mathcal{G}$ is symmetric and nonnegative, but it could be singular. However, the matrix $\operatorname{Diag}\{1 / 2,1 / 2, \ldots, 1 / 2\}+\mathcal{G}$ gives a Riemannian metric on the real $2 n$ dimensional vector space. The factor $1 / 2$ of the Euclidian part is chosen in order that the Euclidian norm of a vector corresponding to a complex n-vector of unit norm is also unity.

Once the diffusion metric $\operatorname{Diag}\{1 / 2,1 / 2, \ldots, 1 / 2\}+\mathcal{G}$ is calculated the standard formulas [18] give the connection coefficients $\Gamma_{\mu \nu}^{k}$ of the Levi-Civita connection for this metric in terms of the coefficients $g_{\mu \nu}=\delta_{\mu \nu} / 2+(\mathcal{G})_{\mu \nu}$ only

$$
\Gamma_{\mu \nu}^{k}=\frac{1}{2} g^{k \lambda}\left(\partial_{\mu} g_{\lambda \nu}+\partial_{\nu} g_{\lambda \mu}-\partial_{\lambda} g_{\mu \nu}\right)
$$

Curvature tensor, Ricci tensor and the scalar curvature of the diffusion metric are also given by the standard formulas [18]:

$$
\begin{aligned}
R_{\lambda \mu \nu}^{k} & =\partial_{\mu} \Gamma_{\nu \lambda}^{k}-\partial_{\nu} \Gamma_{\mu \lambda}^{k}+\Gamma_{\nu \lambda}^{\eta} \Gamma_{\mu \eta}^{k}-\Gamma_{\mu \lambda}^{\eta} \Gamma_{\nu \eta}^{k}, \\
R i c_{\mu \nu} & =R_{\mu \lambda \nu}^{\lambda}, \quad \mathcal{R}=g^{\mu \nu} \operatorname{Ric}_{\mu \nu} .
\end{aligned}
$$


Before we present the results of calculations of the diffusion metric and its curvature for different types of environments, we would like to consider briefly real representation of the QSD equation in the case when the Linblad operators are Hermitian. This includes, for example, the dephasing environment or measurement, or the primary QSD [19], [6] and other fundamental stochastic modifications of the Schroedinger equation [9, [10, [12]. The goal of this digression is to point out to the connection between the general QSD equation (3) and some other stochastic modifications of the Schroedinger equation that have the form of a norm-preserving diffusion equation, and that consequently the construction of the diffusion metrics and its properties are applicable to these equations also. In the case of Hermitian Linblad operators the real representation of (3) assumes a specially simple and illuminating form. Applying the same derivation as from equation (9) to equation (13) one obtains the following:

$$
\begin{aligned}
d p_{i} & =-H_{i j} q_{j} d t+\left(2<L>L_{i j}-\left(L^{2}\right)_{i j}-<L^{2}>\delta_{i j}\right) p_{j} d t \\
& +\frac{1}{\sqrt{2}}\left(L_{i j}-<L>\delta_{i j}\right) p_{j} d W^{R}+\frac{1}{\sqrt{2}}\left(L_{i j}-<L>\delta_{i j}\right) q_{j} d W^{I}
\end{aligned}
$$

where we have, for reasons of simplicity, included only one Linblad operator and the summation over repeated indexes is assumed. Noticing that for an arbitrary linear operator $B$

$$
B_{i j} q_{j}=\delta_{i j} \frac{\partial<B>}{\partial q_{i}}, \quad B_{i j} p_{i}=\delta_{i j} \frac{\partial<B>}{\partial p_{i}}
$$

equation (23) becomes

$$
d p_{i}=-\frac{\partial<H>}{\partial q_{i}} d t+\frac{\partial \Delta^{2} L}{\partial p_{i}} d t+\frac{1}{\sqrt{2}}\left[\frac{\partial<L>}{\partial q_{i}} d W^{R}+\frac{\partial<L>}{\partial p_{i}} d W^{I}\right]
$$

where $\Delta^{2} L=<L^{2}>-<L>^{2}$. There is an analogous equation for $d q_{i}$. The two sets of equations represent a diffusion process on $R^{2 n}$, consisting of the drift given by a Hamiltonian dynamical system on $R^{2 n}$ with the Hamilton's function $<H>$ and the dissipative part determined by $\Delta^{2} L=<L^{2}>-<$ $L>^{2}$ and the diffusion term determined by $\langle L>$. The drift and the diffusion are such that the norm of the vectors in $R^{2 n}$ is preserved. Furthermore, the equations are invariant under a global gauge transformation corresponding to the multiplication of vectors $|\psi\rangle$ by a phase factor. Takeing into the 
account the norm invariance and the global phase symmetry the equations can be written as a diffusion equation on the phase space $S^{2 n-1} / S^{1}$ of the following form

$$
d X=\Omega \nabla<H>d t+\nabla\left(\Delta^{2} L\right) d t+\frac{1}{\sqrt{2}} \nabla<L>d W
$$

where $\nabla$ and $\Omega \nabla$ are the gradient and the skew gradient on $S^{2 n-1} / S^{1}$, and $X$ denotes the set of $2 n-2$ coordinates on the reduced phase space $S^{2 n-1} / S^{1}$. Equations like (25) have been analyzed as candidates for a description of the spontaneous state reduction in [13], or in the case $\hat{L}=\hat{H}$ in [12].

\section{QSD metric and qualitative properties of dynamics}

Application of formula (16) gives for the case (5) of the QSD equation an explicit procedure for calculation of the diffusion metric coefficients $g_{i j}$, in terms of the coefficients of the Linblad operators and the coefficients of the state $\psi>$ in some bases $\left|\psi>=\sum_{i} c_{i}\right| i>$. The components of the diffusion matrix $G=B B^{\dagger}$ are given by

$$
B_{k k^{\prime}}(c, \bar{c})=\sum_{l}^{m} \sum_{j, j^{\prime}}^{n}\left(L^{l}-<L^{l}>\mathbf{1}\right)_{k j}\left(L^{l \dagger}-<L^{l \dagger}>\mathbf{1}\right)_{k^{\prime} j^{\prime}} c_{j} \bar{c}_{j}^{\prime}
$$

where: $\left\langle L^{l}\right\rangle=\sum_{s s^{\prime}} L_{s s^{\prime}} c_{s}^{\prime} \bar{c}_{s}$. Expressing $c_{i}, \bar{c}_{i}$ in terms of $x_{1} \ldots x_{2 n}$

$$
\begin{array}{r}
x_{i}=\left(\bar{c}_{i}+c_{i}\right) / \sqrt{2} \quad i=1, \ldots n \\
x_{i}=\sqrt{-1}\left(\bar{c}_{i}-c_{i}\right) / \sqrt{2} \quad i=n+1, \ldots 2 n,
\end{array}
$$

separating of $G^{R}$ and $G^{I}$ and substituting in (16) finally gives the $4 n^{2}$ entries of the real matrix $\mathcal{G}$.

We shall study the diffusion metric for the following three types of environments: (a) dephasing environment; (b) the environment corresponding to measurement of an observable and (c) thermal environment. The first two are represented by Hermitian and the third one by a non-Hermitian Linblad operators. The main geometrical object which we shall study are the diffusion metric norm of a state vector and its scalar curvature. In order to illustrate how these objects depend on the environment we shall use the simplest but 
important quantum system, namely a single qubit. The system operators can be expressed as combinations of the Pauli sigma matrices $\hat{\sigma}_{x}, \hat{\sigma}_{y}, \hat{\sigma}_{z}$, a state $|\psi\rangle$ of unit norm is determined by $\left\langle\hat{\sigma}_{x}\right\rangle,\left\langle\hat{\sigma}_{y}\right\rangle,\left\langle\hat{\sigma}_{z}\right\rangle$ or by the spherical angles $(\theta, \phi)$ given by

$$
\begin{aligned}
& <\hat{\sigma}_{z}>=\cos (\theta) \\
& <\hat{\sigma}_{x}>=\sin (\theta) \cos (\phi) \\
& <\hat{\sigma}_{y}>=\sin (\theta) \sin (\phi),
\end{aligned}
$$

The environment operators are [20], 3

$$
\hat{L}=\mu \hat{\sigma}_{+} \hat{\sigma}_{-}
$$

for the dephasing and

$$
\hat{L}=\mu_{1} \hat{\sigma}_{+}+\mu_{2} \hat{\sigma}_{-}
$$

for the thermal environment, with $\mu_{1}$ and $\mu_{2}$ proportional to the temperature, and finally for the measurement of, say, $\hat{\sigma}_{z}$ the Linblad operator is just

$$
\hat{L}=\mu \hat{\sigma}_{z} \text {. }
$$

The formulas for the entries $g_{i j}$ of the diffusion metrics in terms of the coordinates $x_{1}, x_{2}, x_{3}, x_{4}$ in the three considered cases can be conveniently written using the following notation:

$$
\begin{aligned}
d_{1}^{2} & =x_{1}^{2}+x_{3}^{2}, \quad d_{2}^{2}=x_{2}^{2}+x_{4}^{2} \\
s & =x_{1} x_{2}+x_{3} x_{4}, \quad a=x_{1} x_{4}-x_{2} x_{3} .
\end{aligned}
$$

Because many of the metric entries are repeated, it is more convenient to present them in a list rather than to write down the corresponding matrices. Using the notation (32), the entries of the metrics in the three considered cases are: For dephasing:

$$
\begin{aligned}
& g_{11}=1 / 2+\left(\mu^{2} / 16\right) d_{1}^{2}\left(2+d_{1}^{2}\right)^{2}, g_{12}=\left(\mu^{2} / 16\right) s d_{1}^{2}\left(2+d_{1}^{2}\right), g_{13}=0 \\
& g_{14}=\left(\mu^{2} / 16\right) a d_{1}^{2}\left(2+d_{1}^{2}\right), g_{22}=1 / 2+\left(\mu^{2} / 16\right) d_{1}^{4} d_{2}^{2}, g_{23}=-g_{14} \\
& g_{24}=0, g_{33}=g_{11}, g_{34}=g_{21}, g_{44}=g_{22} ;
\end{aligned}
$$

For the thermal environment:

$$
\begin{aligned}
& g_{11}=1 / 2+d_{2}^{2}\left[d_{1}^{2} \mu_{1}^{2}+\left(2+d_{1}^{2}\right)^{2} \mu_{2}^{2}\right] / 16 \\
& g_{12}=s\left[d_{1}^{2}\left(2+d_{2}^{2}\right) \mu_{1}+\left(2+d_{1}^{2}\right) d_{2}^{2} \mu_{2}\right] / 16, g_{1,3}=0 \\
& g_{14}=a\left[d_{1}^{2}\left(2+d_{2}^{2}\right) \mu_{1}+\left(2+d_{1}^{2}\right) d_{2}^{2} \mu_{2}\right] / 16, \\
& g_{22}=1 / 2+d_{1}^{2}\left[d_{2}^{2} \mu_{2}^{2}+\left(2+d_{2}^{2}\right)^{2} \mu_{1}^{2}\right] / 16 \\
& g_{13}=0, g_{23}=-g_{14}, g_{24}=0, g_{33}=g_{11}, g_{34}=g_{21}, g_{44}=g_{22} ;
\end{aligned}
$$



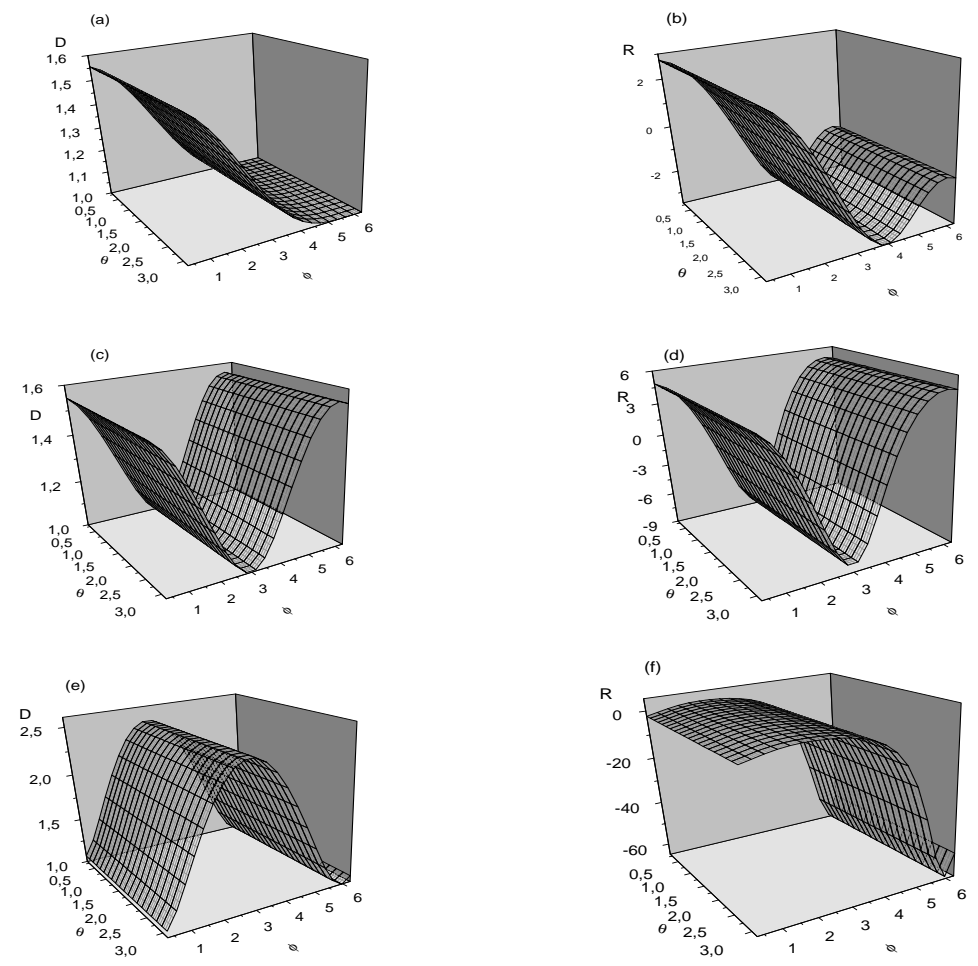

Figure 1: Poincaré sections for the separability constrained non-symmetric quantum dynamics (39). The parameters are $\omega=1, h=1.5$ and (a) $\mu=1.3$, (b) $\mu=1.7$

and for the measurement of $\hat{\sigma}_{z}$

$$
\begin{aligned}
& g_{11}=1 / 2+\left(g^{2} / 16\right) d 1^{2}\left(2+d_{1}^{2}-d_{2}^{2}\right)^{2} \\
& g_{12}=\left(g^{2} / 16\right) s\left(d_{2}^{2}-d_{1}^{2}-2\right)\left(d_{1}^{2}-d_{2}^{2}+2\right), g_{13}=0 \\
& g_{14}=a\left(d_{1}^{2}+d_{2}^{2}+d_{1}^{2} d_{2}^{2}\right), g_{22}=1 / 2+\left(g^{2} / 16\right) d_{2}^{2}\left(2+d_{2}^{2}-d_{1}^{2}\right)^{2} \\
& g_{23}=-g_{14}, g_{24}=0, g_{33}=g_{11}, g_{34}=g_{21}, g_{44}=g_{22} .
\end{aligned}
$$

These formulas are used to compute the diffusion metric norm and the scalar curvature as functions of the state parameters $\theta$ and $\phi$. We shall first consider the dependence of the stated properties of the diffusion metric on the type of the environment and the coupling strengths $\mu, \mu_{1}, \mu_{2}$ and then analyze the relation between these properties and the stochastic dynamics of the state vectors. 

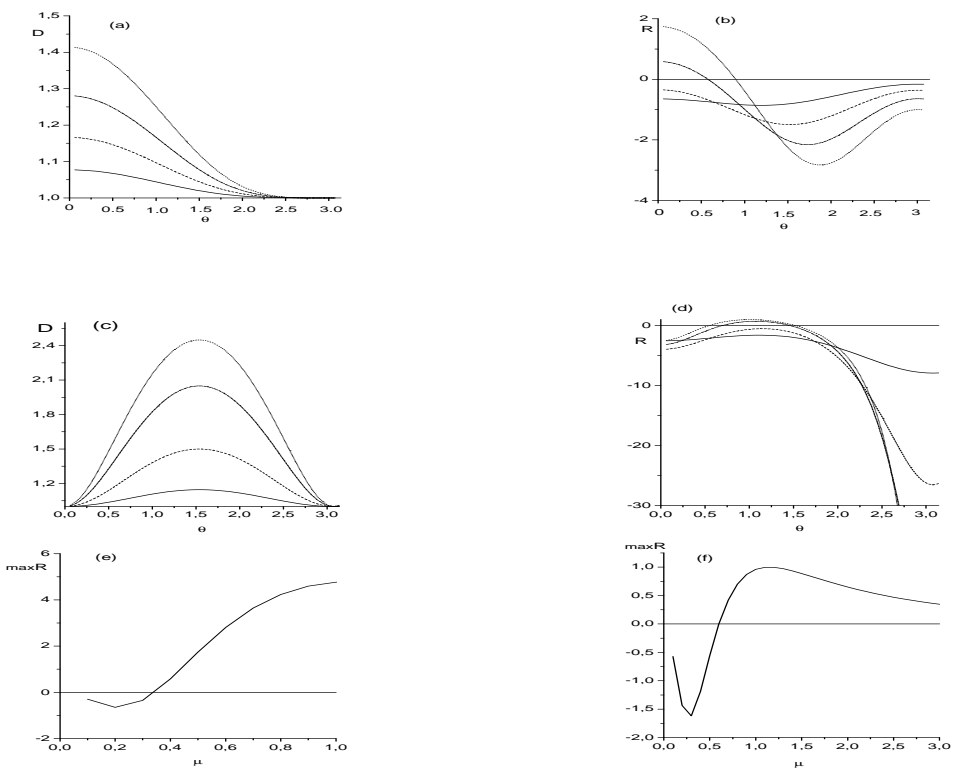

Figure 2: Poincaré sections for the separability constrained non-symmetric quantum dynamics (39). The parameters are $\omega=1, h=1.5$ and (a) $\mu=1.3$, (b) $\mu=1.7$

In Figures 1 and 2 we illustrate the diffusion metric norm and curvature considered as functions on the sphere of states fixed by the type of environment and the value of the corresponding coupling $\mu, \mu_{1}, \mu_{2}$. Consider first Figure 1. The first row (fig. (a),(c),(e)) represent the diffusion metric norm and the second row (fig. (b),(d),(f)) the curvature for the three types of the environments and for some typical fixed values of the corresponding coupling strengths. The curvature is not constant, and can be positive or negative depending on the state vector and on the coupling strength. The maxima of the curvature can be sharp like in the cases of the dephasing and measurement of $\sigma_{z}$. On the other hand, in the thermal case the maxima is surrounded by a large neighborhood of states with almost maximal value of the curvature. Thus, the curvature has a sharp maxima only at the states which are clearly favored by the environment. If there are no such states the curvature maximum differs very little from the neighboring values. The curvature minima are at the states that are like repellers for the environment dominated dynamics.

Dependence of the curvature maxima and the norm on the coupling 
strength is illustrated in Figure 2 for the dephasing and the thermal environments. The most important information from these Figures is that in the dephasing and measurement ( not shown) cases there are clearly sharp values of corresponding coupling strength where the curvature maxima goes from negative to positive values. Also, we see that the curvature minima are negative for all values of the coupling strength.

We shall now study the relation between the sign of the curvature maxima and a stability of the stochastic dynamics of the state vector. The relation will not be analyzed in a mathematically rigorous way using an appropriate notion of the stochastic stability and considering the evolution of the metric as a stochastic process governed by the process $\mid \psi(t)>$. Instead, our strategy is to compute the curvature along different sample paths and see if the path remains near the state corresponding to the curvature maxima. We do such computations for the evolution governed by the environment and an additional fixed small hamiltonian, and we pay special attention to the case when the Linblad operators and the hamiltonian do not commute. The computations are repeated for the values of the coupling to the environment slightly above and below the critical value when the curvature maxima is zero. If the Hamiltonian perturbation is zero the sample paths that started near a maximum of the curvature remain near this maximum. For very small added Hamiltonian part and for a fixed value of the coupling to the environment, the sample paths of the system could wonder away from the maximum or could remain near it. In the former case we shall say that the stochastic dynamics is unstable and in the later case it is stable. The relevant computations are illustrated in Figures 3 and 4.

In the case of the dephasing environment (or the measurement of $\hat{\sigma}_{z}$ ), when the maxima of the curvature are sharply picked, Figures 3 clearly illustrate that positive curvature maxima correspond to the stability and negative to instability in the above mentioned sense. On the other hand, in the thermal case, the dynamics is always unstable even if there is no Hamiltonian perturbation. This is illustrated in Figure 4. We can conclude that the diffusion metric curvature provides us with a clear picture of the qualitative properties of the system's dynamics under strong influence of the environment.

It is well known that if the Linblad operators are Hermitian and commute with the hamiltonian, than the attractors of the stochastic QSD dynamics are the common eigenstates of the Hamiltonian and the Linblad operators [6],[13]. The curvature maxima coincide with the eigenstates of the Linblad 

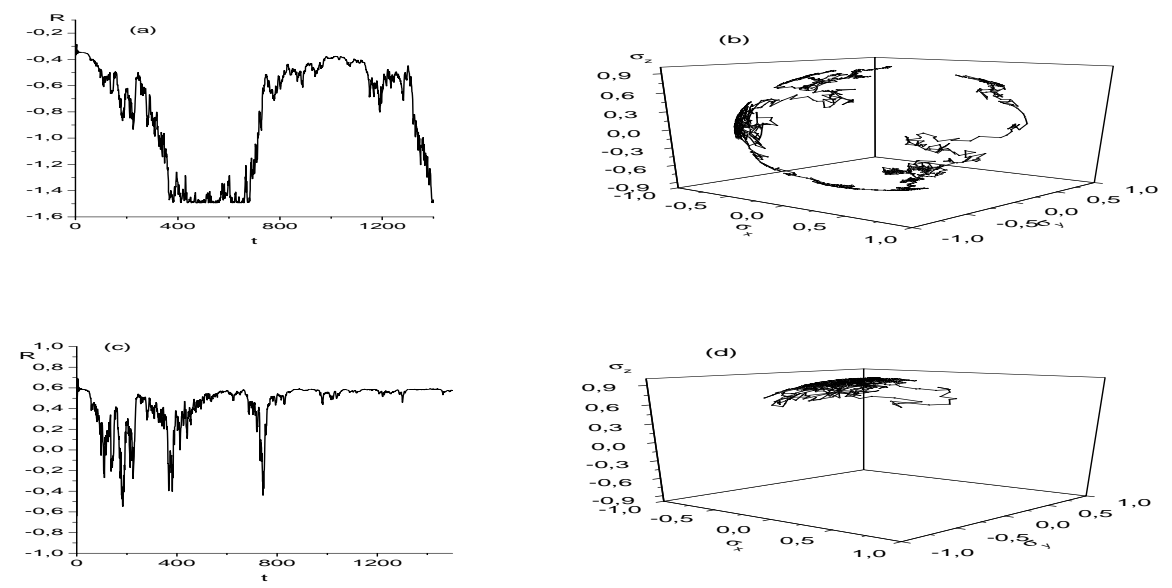

Figure 3: Poincaré sections for the separability constrained non-symmetric quantum dynamics (39). The parameters are $\omega=1, h=1.5$ and (a) $\mu=1.3$, (b) $\mu=1.7$

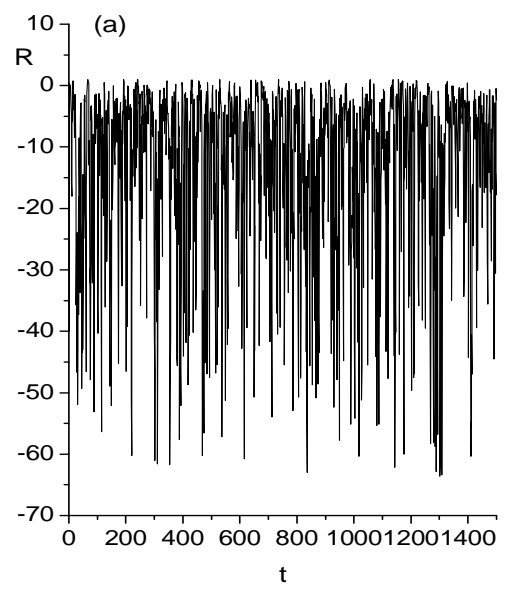

(b)

$\sigma_{z}$

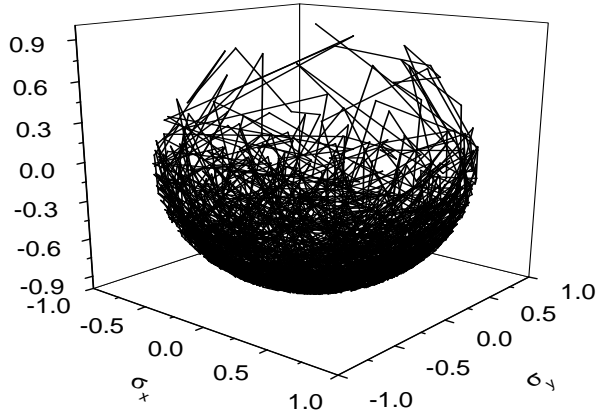

Figure 4: Poincaré sections for the separability constrained non-symmetric quantum dynamics (39). The parameters are $\omega=1, h=1.5$ and (a) $\mu=1.3$, (b) $\mu=1.7$ 
operators, and consequently with the eigenstates of the hamiltonian. The probability of convergence to one of the attractors is, in this case, determined solely by the distance of the initial state from the attractor eigenstate, that is by the quantum mechanical transition probability, and does not depend on the parameters of the Hamiltonian and stochastic terms. The sign of the curvature maxima has no effect on this probability. This is the reason why we expected that the relevance of the sign of the curvature maxima on the stochastic stability is manifested if the Linblad operator and the Hamiltonian perturbation do not commute. This expectation is qualitatively confirmed, as we described and illustrated in Figures 3 and 4, by numerical computations. Observations of numerical sample paths, when the Linblad and the Hamiltonian operators do not commute, are enough to establish the qualitative connection between the maxima of the curvature and the stability of small domains near the maxima.

Finally, our treatment of the relation between the geometry of the diffusion and the stability of the stochastic dynamics is rather heuristic. We treated the diffusion metric as a given field on $R^{2 n}$ (determined by the Linblad operators), and we numerically studied the paths of the stochastic process $\mid \psi(t)>$ in relation to the sign of the curvature maxima. However, the problem of stability versus the properties of the diffusion metric should be formulated and studied using the appropriate notions of stochastic stability [21], [22]. Nevertheless, we think that the numerical evidence strongly indicates that there is a clear relation between the sign and the shape of the curvature maxima and the systems dynamical stability.

\section{Summary and discussion}

According to the view of QSD theory, evolution of a state of an open quantum system is a diffusion process governed by a complex stochastic differential equation on the Hilbert space of the system. The diffusion term of the QSD evolution equation explicitly depends on the operators modelling the environment and on the current state vector of the system. We have studied the Riemannian metric associated with the diffusion term in the QSD equation. The metric is defined on the real $2 \mathrm{n}$-dimensional space (here $\mathrm{n}$ is the complex dimension of the Hilbert space) and is directly related to the properties of the Linblad operators of the environment. We have shown that the scalar curvature of the metric has local maxima at states that are favored 
by the corresponding environment. The curvature at different points, and in particular its local maxima, can be be negative or positive depending on the strength of the coupling to the environment. Also, the sharpness of the curvature maxima reflects the type of the environment. We have shown that there is a sense in which the sign of the curvature maxima is related to the stability of the corresponding state under the addition of a small perturbation that does not commute with the considered Linblad operator. If the environment type and the coupling strength are such that the curvature has sharp positive maxima, than the corresponding state is likely to attract the states of the system whose evolution is governed by the environment and a Hamiltonian that do not necessarily commute. On the other hand, if the curvature maxima are negative, the corresponding states are dynamically unstable under a small Hamiltonian perturbation that does not commute with the Linblad operators. In conclusion, the curvature of the diffusion metric is a relatively easy to calculate, and a very good indicator of what the environment dominated dynamics of the system would look like.

The QSD equation describes the evolution of a pure quantum state using the Hilbert space of the quantum system, but, because it is norm-preserving, it gives also an equation on the state space, namely on the space of rays of the Hilbert space. The Riemannian metric associated with the diffusion on $R^{2 n}$ gives a Hermitian modification of the Fubini-Study metric on $C P^{n-1}$. It is common to consider the complex projective manifold with the associated Fubini-Study metric as the proper framework for the geometry of quantum states [23], 24], so the modification of the metric due to the diffusion should also be formulated within this framework.

The examples that we have analyzed in this paper are restricted on a single qubit under the influence of various types of environments. It would be interesting to analyze the properties of the diffusion metric in the case of coupled gubits, and in particular to see what is the curvature at the entangled states. Probably the proper framework for such analyzes is the formulation on $C P^{n-1}$, mentioned in the previous paragraph, because the entangled states then have characteristic geometric interpretation [23].

Acknowledgements This work is partly supported by the Serbian Ministry of Science contract No. 141003. I should also like to acknowledge the support and hospitality of the Abdus Salam ICTP. 


\section{References}

[1] Lindblad G 1976 Commun. Math. Phys. 48119.

[2] Breuer H-P and Petruccione F 2001 The Theory of Open Quantum Systems ( Oxford: Oxford University Press.)

[3] Carmichael H J 1983. An Open Systems Approach to Quantum Optics (Berlin: Springer-Verlag, Berlin)

[4] Pashkin Yu A et al...2003 Nature 421823

[5] Buric N 2005 Phys.Rev. A 72042322

[6] Percival I C 1999 Quantum State Difussion (Cambridge: Cambridge Uni. Press.)

[7] Belavkin V P 1999 Rep.Math.Phys. 43405

[8] Gardiner C W and Zoller P 2000 Quantum Noise ( Berlin: SpringerVerlag)

[9] Pearle P 1993 Phys.Rev.A 48913

[10] Bassi A and Chirardi G 2003 Phys.Rep. 379257

[11] Gisin N 1989 Helv.Phys.Acta 62363

[12] Hughston L P 1996 Proc.R.Soc.Lond. A 452953

[13] Adler S L and Brun T A 2001 J.Phys.A: Math. Gen. 344797

[14] Schack R, Brun T A and Percival I C 1996. Phys.Rev.A. 532696

[15] Wiseman H M and Milburn G Phys. Rev.A 47642

[16] Molmer K, Castin Y and Dalibar J 1993 J. Opt. Soc. Am. B 10524

[17] Burić N 2006 Phys.Rev.A 73052111

[18] Kobajayashi S and Nomizu K 1969 Foundations of Differential Geometry ( New York: Wiley)

[19] Percival I C 1995 Proc.R.Soc. A 451503 
[20] Mintert F, Carvalho A R, Kus M and Buchleitner A 2005 Phys. Rep. 415207

[21] Khas'minski R Z 1980 Stochastic Stability of Differential Equations (Alphen aan der Rijn: Sijthoff and Noordhoff)

[22] Arnold L 1998 Random Dynamical Systems ( Berlin: Springer Verlag)

[23] Brody D C and Hughston L P 2001 J.Geom. Phys. 3819

[24] Bengtsson I and Žyczkowski K 2006 Geometry of Quantum States (Cambridge: Cambridge University Press) 


\section{FIGURE CAPTIONS}

Figure 1 Diffusion metric norm (a,c,e) and curvature (b,d,f) as functions of state parametrized by $(\theta, \phi)$, for dephasing environment with $\mu=0.6$ (a,b); measurement of $\hat{\sigma}_{z}$ with $\mu=1$. (c,d) and thermal environment with $\mu_{1}=2, \mu_{2}=1(\mathrm{e}, \mathrm{f})$.

Figure 2 Diffusion metric norm (a,c) and curvature (b,d) as functions of $\theta$ for different values of the parameters $\mu$ or $\mu_{1}, \mu_{2}$, and the maximum over $(\theta, \phi)$ of the curvature as a function of $\mu$ (e) or $\mu_{1}=2 \mu_{2}=2 \mu(f)$. Figures a,b,e corespond to the dephasing and $c, d, f$ to the thermal environment.

Figure 3 Diffusion metric curvature (a,c) along the corresponding stochastic path illustrated in b,d for the dephasing environment and $\mu=0.3$ when $\max R<0(\mathrm{a}, \mathrm{b})$, and $\mu=0.5$ when $\max R>0(\mathrm{c}, \mathrm{d})$. The small Hamiltonian perturbation is $0.01 \hat{\sigma}_{x}$.

Figure 4 Diffusion metric curvature (a) along a stochastic path (b) for the thermal environment and $\mu_{1}=2 \mu_{2}=1.6$. The Hamiltonian part is zero. 